PROCEEDINGS OF THE

AMERICAN MATHEMATICAL SOCIETY

Volume 131, Number 2, Pages 563-576

S 0002-9939(02)06565-6

Article electronically published on May 29, 2002

\title{
ON THE BOUNDEDNESS OF HAMILTONIAN OPERATORS
}

\author{
TOMAS YA. AZIZOV, AAD DIJKSMA, AND IRINA V. GRIDNEVA
}

(Communicated by Joseph A. Ball)

\begin{abstract}
We show that a non-negative Hamiltonian operator whose domain contains a maximal uniformly positive subspace is bounded.
\end{abstract}

\section{INTRODUCTION}

Let $\{\mathcal{G},(\cdot, \cdot)\}$ be a Hilbert space and consider the orthogonal direct sum

$$
\mathcal{H}=\mathcal{G} \oplus \mathcal{G},
$$

which is a Hilbert space whose inner product we also denote by $(\cdot, \cdot)$. A bounded operator $\mathfrak{A}$ on $\mathcal{H}$ is called a Hamiltonian operator if with respect to the decomposition (1) it has the $2 \times 2$ block matrix representation

$$
\mathfrak{A}=\left[\begin{array}{cc}
A & B \\
C & -A^{*}
\end{array}\right]
$$

where $B$ and $C$ are self-adjoint operators on $\mathcal{G}$. If additionally $B$ and $C$ are both non-negative, then $\mathfrak{A}$ is called a non-negative Hamiltonian operator. In the space $\mathcal{H}$ we consider the block matrices

$$
J=\left[\begin{array}{cc}
0 & I \\
I & 0
\end{array}\right], \quad \mathfrak{J}=\left[\begin{array}{cc}
0 & i I \\
-i I & 0
\end{array}\right]
$$

and introduce two Krein spaces (for the definition see (i) below) $\mathcal{K}_{J}:=\left\{\mathcal{H},[\cdot, \cdot]_{J}\right\}$ and $\mathcal{K}_{\mathfrak{J}}:=\left\{\mathcal{H},[\cdot, \cdot]_{\mathfrak{J}}\right\}$ whose indefinite inner products are defined by

$$
[\cdot, \cdot]_{J}=(J \cdot, \cdot), \quad[\cdot, \cdot]_{\mathfrak{J}}=(\mathfrak{J} \cdot, \cdot),
$$

respectively. Evidently, the bounded operator $\mathfrak{A}$ on the Hilbert space $\mathcal{H}=\mathcal{G} \oplus \mathcal{G}$ is Hamiltonian if and only if $i \mathfrak{A}$ is self-adjoint in the Krein space $\mathcal{K}_{\mathfrak{J}}$, and $\mathfrak{A}$ is a non-negative Hamiltonian operator if it is a Hamiltonian operator such that $i \mathfrak{A}$ is dissipative (for the definition see (ii) below) in the Krein space $\mathcal{K}_{J}$. The extension of these definitions to the case of an unbounded operator $\mathfrak{A}$ is now evident: A closed densely defined operator $\mathfrak{A}$ on $\mathcal{H}=\mathcal{G} \oplus \mathcal{G}$ will be called Hamiltonian if $i \mathfrak{A}$ is self-adjoint in the Krein space $\mathcal{K}_{\mathfrak{J}}$ and $\mathfrak{A}$ will be called a non-negative Hamiltonian operator if it is Hamiltonian and $i \mathfrak{A}$ is dissipative in the Krein space $\mathcal{K}_{J}$. The aim of this note is to give conditions which imply the boundedness of Hamiltonian operators. The main results in this paper, Theorems 5 and 6 , show that non-negative

Received by the editors March 13, 2001 and, in revised form, September 28, 2001.

2000 Mathematics Subject Classification. Primary 47B50, 46C20, 47B44, 47B25.

This research was supported by grants NWO 047-008-008 and RFBR 99-01-00391. 
Hamiltonian operators are bounded if they satisfy the condition (L) in one of the two Krein spaces $\mathcal{K}_{J}$ and $\mathcal{K}_{\mathfrak{J}}$.

To formulate property (L) and to prove the theorems, we use results from the theory of operators on Krein spaces; see, for example, $[\mathrm{AI}]$, [DR]. In the following paragraphs we briefly recall (i) the definition of a Krein space and some of its properties, (ii) the definition of a (maximal, uniformly) dissipative operator on a Krein space, and (iii) the condition (L). This will also make clear the notation we use in the sequel.

(i) A Krein space $\{\mathcal{K},[\cdot, \cdot]\}$ is a linear space $\mathcal{K}$ over the complex numbers $\mathbb{C}$ equipped with an indefinite inner product $[\cdot, \cdot]$ such that $\mathcal{K}$ admits a decomposition

$$
\mathcal{K}=\mathcal{K}^{+} \oplus \mathcal{K}^{-},
$$

in which the summands $\left\{\mathcal{K}^{+},[\cdot, \cdot]\right\}$ and $\left\{\mathcal{K}^{-},-[\cdot, \cdot]\right\}$ equipped with the indicated inner products are Hilbert spaces and $\left[\mathcal{K}^{+}, \mathcal{K}^{-}\right]=\{0\}$. Note that the inner product is non-degenerate in the sense that if for some $x \in \mathcal{K}$ it holds that $[x, \mathcal{K}]=\{0\}$, then $x=0$. The decomposition (2) is called a fundamental decomposition. It gives rise to a Hilbert space inner product, namely

$$
(x, y):=\left[x^{+}, y^{+}\right]-\left[x^{-}, y^{-}\right], \quad x=x^{+}+x^{-}, y=y^{+}+y^{-}, \quad x^{ \pm}, y^{ \pm} \in \mathcal{K}^{ \pm} .
$$

The corresponding Hilbert space norm $\|\cdot\|$ depends on the fundamental decomposition, but any two such decompositions give rise to equivalent norms. Topological notions such as convergence, closure, and boundedness are always considered with respect to any one of these norms. A closed linear manifold in a Krein space $\mathcal{K}$ will be called a subspace. By $L(\mathcal{K})$ we denote the set of bounded operators on $\mathcal{K}$. The identity operator will be designated by $I$; it should be clear from the context on which space it acts. The adjoint $A^{*}$ of a bounded or densely defined operator $A$ on a Krein space $\mathcal{K}$ and the orthogonal complement $\mathcal{M}^{\perp}$ of a subspace $\mathcal{M}$ of $\mathcal{K}$ are defined with respect to the indefinite inner product on $\mathcal{K}$ in the same way as if defined on a Hilbert space. A subspace $\mathcal{M}$ is called regular in $\{\mathcal{K},[\cdot, \cdot]\}$ if $\{\mathcal{M},[\cdot, \cdot]\}$ is a Krein space.

If $\{\mathcal{K},[\cdot, \cdot]\}$ is a Krein space and (2) holds, there is a bounded operator $J$ on $\mathcal{K}$, called the fundamental symmetry corresponding to (2), such that $[\cdot, \cdot]=(J \cdot, \cdot)$ and it is easy to see that $J$ is both self-adjoint and unitary with respect to $(\cdot, \cdot)$ as well as with respect to $[\cdot, \cdot]$. Moreover, the operators

$$
P^{+}=\frac{1}{2}(I+J), \quad P^{-}=\frac{1}{2}(I-J)
$$

are self-adjoint projections in $\mathcal{K}$ onto $\mathcal{K}^{+}$and $\mathcal{K}^{-}$, respectively. It is natural to construct a Krein space from a Hilbert space $\{\mathcal{H},(\cdot, \cdot)\}$ and a bounded self-adjoint operator $W$, say, on $\mathcal{H}$ by introducing the indefinite scalar product $[\cdot, \cdot]_{W}=(W \cdot, \cdot)$. The space $\left\{\mathcal{H},[\cdot, \cdot]_{W}\right\}$ is called a $W$-space. This construction is too general to generate a Krein space: A $W$-space is a Krein space if and only if $W$ is boundedly invertible. If this condition holds, then a fundamental decomposition of the space is provided by the spectral subspaces of $W$ related to the intervals $(0, \infty)$ and $(-\infty, 0)$. From the definition of a Krein space it follows that each Krein space is a $J$-space with respect to some fundamental decomposition.

A subspace $\mathcal{M}$ of a Krein space $\{\mathcal{K},[\cdot, \cdot]\}$ is called non-negative if $[x, x] \geq 0$ for all $x \in \mathcal{M}$, positive if $[x, x]>0$ for all $x \in \mathcal{M} \backslash\{0\}$, and uniformly positive if with respect to the norm $\|\cdot\|$ associated with one of the fundamental decompositions and for some $\varepsilon>0$ it holds that $[x, x] \geq \varepsilon\|x\|$ for all $x \in \mathcal{M}$. Non-positive, negative, and 
uniformly negative subspaces are defined similarly. A subspace $\mathcal{M}$ is called (semi-) definite if $\mathcal{M}$ is (non-)positive or (non-)negative and it is called neutral if $[x, x]=0$ for all $x \in \mathcal{M} . \mathcal{M}$ is called maximal with respect to any of these properties if it has this property and is not properly contained in a subspace having this property also.

(ii) Let $\{\mathcal{K},[\cdot, \cdot]\}$ be a Krein space. A closed densely defined operator $A$ is called dissipative if $\operatorname{Im}[A x, x] \geq 0$ for all $x \in \operatorname{dom} A$. If with respect to the norm $\|\cdot\|$ associated with one of the fundamental decompositions and for some $\varepsilon>0$ it holds that $\operatorname{Im}[A x, x] \geq \varepsilon\|x\|^{2}$ for all $x \in \operatorname{dom} A$, then $A$ is called a uniformly dissipative operator. It is a maximal (uniformly) dissipative operator if it is (uniformly) dissipative and admits no non-trivial (uniformly) dissipative extensions. In the sequel we shall use a lemma from Shkalikov $[\mathrm{S}]$ which describes some spectral properties of maximal uniformly dissipative operators on a Krein space which have the property (L); see Lemma 1 in the next section.

(iii) Following [AI, Definition 3.1.5], we say that a densely defined operator $A$ on a Krein space $\mathcal{K}$ satisfies the condition (L) and write $A \in(\mathrm{L})$, if $\operatorname{dom} A$ contains a maximal uniformly positive subspace. Evidently, this maximal uniformly positive subspace contained in $\operatorname{dom} A$ is the $\mathcal{K}^{+}$in some fundamental decomposition of $\mathcal{K}$. The condition (L) was introduced by Langer in [L1], [L2], in theorems on the existence of invariant subspaces for self-adjoint operators in a Krein space. This condition is very natural in certain problems from mechanics. Indeed, we recall (see, for example, $\mathrm{KL}$ ) that many of these can be reduced to problems for an operator pencil of the form

$$
L(\lambda)=\lambda^{2}+\lambda B+C
$$

in which $B$ is a maximal dissipative operator and $C$ is a bounded positive selfadjoint operator on a Hilbert space $\mathcal{F}$. If we introduce $\mathcal{K}=\mathcal{F} \oplus \mathcal{F}$ and consider it as a $J$-space with

$$
J=\left[\begin{array}{cc}
I & 0 \\
0 & -I
\end{array}\right]
$$

then the operator

$$
\mathfrak{C}=\left[\begin{array}{cc}
0 & C^{1 / 2} \\
-C^{1 / 2} & -B
\end{array}\right]
$$

is maximal dissipative, satisfies the condition (L) and describes the spectral properties of the pencil (3). If $B$ is unbounded, then so is $\mathfrak{C}$. The condition (L) is also used in, for example, the paper [LT] on boundary eigenvalue problems with boundary conditions which depend rationally on the eigenvalue. Finally, we mention that the condition (L) appears in a boundedness criterion for densely defined expansive operators on a Krein space of Shmul'yan [Shm; it is a generalization of an earlier result of Brodskii [B]. For a recent treatment of Shmul'yan's theorem, see [DR].

Section 1 contains four preliminary results including Shkalikov's Lemma mentioned in (ii) above. In Sections 2 and 3 we prove the main results of this paper, Theorems 5 and 6 . In Section 4 we give two examples of Hamiltonian operators which are unbounded. In Example 7 the operator is non-negative but does not have property (L). In Example 8 the operator has property $(\mathrm{L})$ but is not non-negative.

In the sequel we denote by $\sigma(A)$ and $\rho(A)$ the spectrum and the resolvent set of the operator $A$, respectively, by $\mathbb{R}$ the set of real numbers, and by $\mathbb{C}^{ \pm}$the open upper/lower half plane in the set of complex numbers $\mathbb{C}$. We use ${ }^{*}$ to denote the 
adjoint of an operator relative to the inner products defined on the spaces on which the operator acts and the complex conjugate of a complex number. If $\sigma \subset \mathbb{C}$, then $\sigma^{*}=\left\{\lambda^{*} \mid \lambda \in \sigma\right\}$.

In $\mathrm{AKK}$ the problem is considered under what conditions a continuous function $\mathfrak{A}:[0,1] \rightarrow L(\mathcal{H})$ whose values are bounded non-negative Hamiltonian operators admits a spectral diagonalization. This means there exist an orthogonal decomposition $\mathcal{H}=\mathcal{H}_{r} \oplus \mathcal{H}_{\ell}$ and a continuous, bounded and boundedly invertible operator function $V:[0,1] \rightarrow L(\mathcal{H})$ such that for each $t \in[0,1], \mathcal{H}_{r}$ and $\mathcal{H}_{\ell}$ are invariant under $\mathfrak{B}(t):=V(t)^{-1} \mathfrak{A}(t) V(t), \sigma\left(\left.\mathfrak{B}(t)\right|_{\mathcal{H}_{r}}\right)$ belongs to the open right half and $\sigma\left(\left.\mathfrak{B}(t)\right|_{\mathcal{H}_{\ell}}\right)$ belongs to the open left half of the complex plane. We plan to extend this theory to functions whose values are closed densely defined non-negative Hamiltonian operators.

\section{Preliminaries}

Here we give four lemmas, which will play a role in our proofs of the main results, Theorems [5] and 6] below. The first one is due to Shkalikov [S].

Lemma 1. Let $A$ be a maximal uniformly dissipative operator in a Krein space $\mathcal{K}$ and assume $A \in(\mathrm{L})$. Then $\rho(A)$,

(i) $\rho(A)$ contains a strip parallel to $\mathbb{R}$ with $\mathbb{R}$ in its interior, in particular $\mathbb{R} \subset$

(ii) $\sigma_{+}(A):=\sigma(A) \cap \mathbb{C}^{+}$is a bounded set, and

(iii) the subspace $\mathcal{L}_{+}:=P_{+}(A) \mathcal{K}$ is maximal uniformly positive in $\mathcal{K}, \mathcal{L}_{+} \subset$ $\operatorname{dom} A$, and $A \mathcal{L}_{+} \subset \mathcal{L}_{+}$, where $P_{+}(A)$ is the Riesz projection for $A$ related to $\sigma_{+}(A)$.

Recall that the Riesz projection is defined by the integral

$$
P_{+}(A)=\frac{1}{2 \pi i} \int_{\Gamma_{+}\left(\sigma_{+}(A)\right)}(\lambda-A)^{-1} d \lambda,
$$

where $\Gamma_{+}\left(\sigma_{+}(A)\right)$ is a positively oriented contour in $\mathbb{C}$ around $\sigma_{+}(A)$ with $\sigma(A) \backslash$ $\sigma_{+}(A)$ in its exterior.

Lemma 2. Let $\mathcal{K}_{1}$ and $\mathcal{K}_{2}$ be two Krein spaces with $\operatorname{dim} \mathcal{K}_{1}=\operatorname{dim} \mathcal{K}_{2}$. For $j=1,2$, let $\mathcal{L}_{j}$ and $\mathcal{M}_{j}$ be neutral subspaces in $\mathcal{K}_{j}$ such that

$$
\mathcal{K}_{j}=\mathcal{L}_{j}+\mathcal{M}_{j}
$$

Then there is a unitary operator $V: \mathcal{K}_{1} \rightarrow \mathcal{K}_{2}$ such that $V \mathcal{L}_{1}=\mathcal{L}_{2}$ and $V \mathcal{M}_{1}=$ $\mathcal{M}_{2}$.

Proof. Denote the indefinite inner product on $\mathcal{K}_{j}$ by $[\cdot, \cdot]_{j}, j=1,2$. Note that (4) is a direct sum, that is, $\mathcal{L}_{j} \cap \mathcal{M}_{j}=\{0\}$. Indeed, if $x \in \mathcal{L}_{j} \cap \mathcal{M}_{j}$, then, because $\mathcal{L}_{j}$ and $\mathcal{M}_{j}$ are neutral,

$$
\left[x, \mathcal{K}_{j}\right]_{j}=\left[x, \mathcal{L}_{j}\right]_{j}+\left[x, \mathcal{M}_{j}\right]_{j}=\{0\}
$$

and $\mathcal{K}_{j}$ being non-degenerate, this implies $x=0$. For $j=1,2$, we consider in $\mathcal{K}_{j}$ any Hilbert space inner product $(\cdot, \cdot)_{j}$ such that $\mathcal{L}_{j}$ is orthogonal to $\mathcal{M}_{j}$. By a theorem of $\mathrm{S}$. Banach this inner product then is equivalent to the inner products generated by the fundamental decompositions on $\mathcal{K}_{j}$. Therefore there is a selfadjoint operator $W_{j}$ such that $[\cdot, \cdot]_{j}=\left(W_{j} \cdot, \cdot\right)_{j}$. The matrix representation of $W_{j}$ 
with respect to the decomposition (4) takes the form

$$
W_{j}=\left[\begin{array}{cc}
0 & S_{j} \\
S_{j}^{*} & 0
\end{array}\right]
$$

where $S_{j}: \mathcal{M}_{j} \rightarrow \mathcal{L}_{j}$ is a bounded and boundedly invertible operator. The fact that (4) is a direct sum and the equality $\operatorname{dim} \mathcal{K}_{1}=\operatorname{dim} \mathcal{K}_{2} \operatorname{imply} \operatorname{dim} \mathcal{L}_{1}=\operatorname{dim} \mathcal{L}_{2}=$ $\operatorname{dim} \mathcal{M}_{1}=\operatorname{dim} \mathcal{M}_{2}$, and hence there exists a bounded and boundedly invertible operator $V_{21}: \mathcal{L}_{1} \rightarrow \mathcal{L}_{2}$. Now the block operator

$$
V=\left[\begin{array}{cc}
V_{21} & 0 \\
0 & S_{2}^{-1}\left(V_{21}^{*}\right)^{-1} S_{1}
\end{array}\right]
$$

has the desired properties.

Lemma 3. Let $A$ be a maximal dissipative operator in a Krein space. If $A \in(\mathrm{L})$, then also $A^{*} \in(\mathrm{L})$.

Proof. Denote the Krein space by $\{\mathcal{K},[\cdot, \cdot]\}$. Without loss of generality we can assume that the fundamental decomposition (2) holds with $\mathcal{K}^{+} \subset \operatorname{dom} A$. Norms will be computed with respect to this decomposition. The orthogonal projections onto $\mathcal{K}^{ \pm}$will be denoted by $P^{ \pm}$. We consider the spaces $\mathcal{K}^{ \pm}$as Hilbert spaces equipped with the inner products $\pm[\cdot, \cdot]$. Let

$$
A=\left[\begin{array}{ll}
A_{11} & A_{12} \\
A_{21} & A_{22}
\end{array}\right]
$$

be the block matrix representation of $A$ with respect to the decomposition (2). As $A$ is closed, the two block columns are closed operators. Because $\mathcal{K}^{+} \subset \operatorname{dom} A$ and on account of the closed graph theorem, $A_{11}$ on $\mathcal{K}^{+}$and $A_{21}: \mathcal{K}^{+} \rightarrow \mathcal{K}^{-}$ are bounded everywhere defined operators. The operators $A_{12}$ and $A_{22}$ have the same domain and are densely defined, but $A_{12}$ may or may not be closed. By AI. Theorem 2.2.9 and Corollary 2.2.12]:

$(\alpha)$ The operator $-A_{22}$ is a closed densely defined maximal dissipative operator in the Hilbert space $\mathcal{K}^{-}$and hence $\mathbb{C}^{+} \subset \rho\left(A_{22}\right)$.

( $\beta$ ) For $\lambda \in \rho\left(A_{22}\right)$, the operator $A_{12}\left(A_{22}-\lambda\right)^{-1}: \mathcal{K}^{-} \rightarrow \mathcal{K}^{+}$is a bounded everywhere defined operator.

We give different, more direct, proofs. To see $(\alpha)$, consider the equality

$$
A\left[\begin{array}{cc}
I & 0 \\
0 & -I
\end{array}\right]=B+\left[\begin{array}{cc}
0 & A_{21}^{*} \\
A_{21} & 0
\end{array}\right], \quad B:=\left[\begin{array}{cc}
A_{11} & -A_{12}-A_{21}^{*} \\
0 & -A_{22}
\end{array}\right] .
$$

In the Hilbert space $\widetilde{\mathcal{H}}:=\left\{\mathcal{K}^{+},[\cdot, \cdot]\right\} \oplus\left\{\mathcal{K}^{-},-[\cdot, \cdot]\right\}$ the operator on the left of the equality sign is maximal dissipative and the second summand on the right is self-adjoint and bounded, and this implies that $B$ is maximal dissipative in $\widetilde{\mathcal{H}}$ also. Thus $\operatorname{ran}(B+i I)=\widetilde{\mathcal{H}}$, and therefore $\operatorname{ran}\left(-A_{22}+i I\right)=\mathcal{K}^{-}$. This equality and the fact that $-A_{22}=-P^{-} A P^{-}$is dissipative in the Hilbert space $\mathcal{K}^{-}$imply $(\alpha)$.

To prove $(\beta)$ we note that $\rho\left(A_{11}\right) \cap \rho\left(-A_{22}\right) \cap \rho(B) \neq \emptyset$. For $\lambda_{0}$ in this intersection, the resolvent of $B$ can be written as

$$
\left(B-\lambda_{0}\right)^{-1}=\left[\begin{array}{cc}
\left(A_{11}-\lambda_{0}\right)^{-1} & \left(A_{11}-\lambda_{0}\right)^{-1}\left(A_{12}+A_{21}^{*}\right)\left(-A_{22}-\lambda_{0}\right)^{-1} \\
0 & \left(-A_{22}-\lambda_{0}\right)^{-1}
\end{array}\right] .
$$

Since the block operator is bounded, the operator in the upper right corner is also bounded. Subtracting the bounded operator $\left(A_{11}-\lambda_{0}\right)^{-1} A_{21}^{*}\left(-A_{22}-\lambda_{0}\right)^{-1}$ from this operator, we are left with the bounded operator $\left(A_{11}-\lambda_{0}\right)^{-1} A_{12}\left(-A_{22}-\lambda_{0}\right)^{-1}$. 
As $A_{11}-\lambda_{0}$ is bijective, we conclude that for $\mu_{0}=-\lambda_{0} \in \rho\left(A_{22}\right)$, the operator $A_{12}\left(A_{22}-\mu_{0}\right)^{-1}$ is bounded. The resolvent identity

$$
\left(A_{22}-\lambda\right)^{-1}=\left(A_{22}-\mu_{0}\right)^{-1}+\left(\lambda-\mu_{0}\right)\left(A_{22}-\mu_{0}\right)^{-1}\left(A_{22}-\lambda\right)^{-1}, \lambda \in \rho\left(A_{22}\right),
$$

now readily implies $(\beta)$.

By [AI, Theorem 2.2.9], if $\operatorname{Im} \lambda_{0}>2\left\|A P^{+}\right\|$, then $\lambda_{0} \in \rho(A)$. According to $(\alpha)$, then also $\lambda_{0} \in \rho\left(A_{22}\right)$. In the proof of [AI Theorem 3.1.13] it is shown that there exists a $\lambda_{0}$ with $\operatorname{Im} \lambda_{0}>2\left\|A P^{+}\right\|$such that

$$
\left\|A_{12}\left(A_{22}-\lambda_{0}\right)^{-1}\right\|<1 \text {. }
$$

For this choice of $\lambda_{0}$, the operator $A-\lambda_{0}$ admits the representation

$$
A-\lambda_{0}=\left[\begin{array}{cc}
A_{11}-\lambda_{0} & A_{12}\left(A_{22}-\lambda_{0}\right)^{-1} \\
A_{21} & I
\end{array}\right] \times\left[\begin{array}{cc}
I & 0 \\
0 & A_{22}-\lambda_{0}
\end{array}\right]
$$

and then $\left(A-\lambda_{0}\right)^{*}$ takes the form

$$
\left(A-\lambda_{0}\right)^{*}=\left[\begin{array}{cc}
I & 0 \\
0 & \left(A_{22}-\lambda_{0}\right)^{*}
\end{array}\right] \times\left[\begin{array}{cc}
\left(A_{11}-\lambda_{0}\right)^{*} & -A_{21}^{*} \\
-\left(A_{12}\left(A_{22}-\lambda_{0}\right)^{-1}\right)^{*} & I
\end{array}\right] .
$$

From the appearance of the operators in the second row of the second matrix and the identity operator in the first matrix on the right-hand side of the last equality we conclude that the subspace

$$
\mathcal{M}:=\left\{x=\left[\begin{array}{c}
x^{+} \\
\left(A_{12}\left(A_{22}-\lambda_{0}\right)^{-1}\right)^{*} x^{+}
\end{array}\right] \mid x^{+} \in \mathcal{K}^{+}\right\}
$$

is contained in $\operatorname{dom} A^{*}$. It remains to note that because of (6), $\mathcal{M}$ is maximal uniformly positive in $\mathcal{K}$.

Lemma 4. Let $A=A^{*}$ be a self-adjoint operator in a Krein space $\mathcal{K}$. Assume $\sigma(A) \cap \mathbb{R}=\emptyset$ and $\sigma_{+}(A)=\sigma(A) \cap \mathbb{C}^{+}$is a bounded set. Let $P_{+}(A)$ be the Riesz projection related to $\sigma_{+}(A)$. Then $P_{+}(A) \mathcal{K}$ is a maximal neutral subspace if and only if $A$ is bounded.

Since the spectrum of a self-adjoint operator on a Krein space is symmetric with respect to the real axis, the assumptions of the lemma imply that $\sigma(A)$ is a bounded set. In a Hilbert space this implies that $A$ is bounded. A proof uses that a selfadjoint operator on a Hilbert space has a non-empty spectrum. (Indeed, if $P_{\sigma}$ is the Riesz projection related to $\sigma(A)$, then $\left.A\right|_{\text {ker } P_{\sigma}}$ is a self-adjoint operator with an empty spectrum which implies ker $P_{\sigma}=\{0\}$, that is, $P_{\sigma}=I$. From the inclusion $\operatorname{ran} P_{\sigma} \subset \operatorname{dom} A$ it then follows that $A$ is everywhere defined and hence bounded.) Let $T$ be a Volterra operator on a Hilbert space $\mathcal{G}$ with $\sigma(T)=\sigma_{c}(T)=\{0\}$. Then the operator

$$
A=\left[\begin{array}{cc}
T^{-1} & 0 \\
0 & T^{-*}
\end{array}\right]
$$

is self-adjoint in the Krein space $\mathcal{K}_{J}$, defined in the beginning of the introduction, has an empty spectrum, but it is unbounded. This example clarifies why maximality is needed in the lemma.

Proof. Assume $A$ is bounded. Then the assumption $\sigma_{+}(A)=\sigma(A) \cap \mathbb{C}^{+}$is a bounded set automatically holds; it is assumed in order to define the Riesz projection $P_{+}(A)$. But now also $\sigma_{-}(A)=\sigma(A) \cap \mathbb{C}^{-}$is a bounded set and hence 
the Riesz projection $P_{-}(A)$ related to $\sigma_{-}(A)$ is well defined. Since $\sigma(A) \cap \mathbb{R}=\emptyset$, $P_{+}(A)+P_{-}(A)=I$, that is,

$$
\mathcal{K}=P_{+}(A) \mathcal{K}+\left(I-P_{+}(A)\right) \mathcal{K}, \quad \text { direct sum. }
$$

On account of [AI, Corollary 2.3.11], $P_{+}(A) \mathcal{K}$ and $\left(I-P_{+}(A)\right) \mathcal{K}$ are neutral subspaces of $\mathcal{K}$, and, by [AI, Proposition 1.1.25], $P_{+}(A) \mathcal{K}$ is a maximal neutral subspace.

We now prove the "only if" part. Let $\sigma=\sigma_{+}(A) \cup \sigma_{+}(A)^{*}$ and let $P_{\sigma}=$ $P_{\sigma}(A)$ be the Riesz projection for $A$ related to $\sigma$. Since $A$ is self-adjoint, $P_{\sigma} \mathcal{K}$ is a regular $A$-invariant subspace, $\left(I-P_{\sigma}\right) \mathcal{K}$ has the same properties, and $\mathcal{K}$ admits the decomposition (see [AI, Corollary 2.3.12 ])

$$
\mathcal{K}=P_{\sigma} \mathcal{K}[+]\left(I-P_{\sigma}\right) \mathcal{K},
$$

where the summands are mutually orthogonal relative to the indefinite inner product $[\cdot, \cdot]$ on $\mathcal{K}$. Since $P_{+}(A) \mathcal{K}$ is a maximal neutral subspace and hence maximal non-positive or maximal non-negative in $\mathcal{K}$, its orthogonal complement is maximal non-negative or maximal non-positive, respectively. From the inclusion $P_{+}(A) \mathcal{K} \subset P_{\sigma} \mathcal{K}$ we have that this orthogonal complement contains the regular subspace $\left(I-P_{\sigma}\right) \mathcal{K}$, which is therefore a uniformly definite subspace. This implies that $\left.A\right|_{\left(I-P_{\sigma}\right) \mathcal{K}}$ is a self-adjoint operator in a Hilbert space and consequently, $\sigma\left(\left.A\right|_{\left(I-P_{\sigma}\right) \mathcal{K}}\right) \cap \mathbb{R} \neq \emptyset$, which, if $\left(I-P_{\sigma}\right) \mathcal{K} \neq\{0\}$, contradicts the assumption $\sigma(A) \cap \mathbb{R}=\emptyset$. Thus we have $\left(I-P_{\sigma}\right) \mathcal{K}=\{0\}$ and hence $A=\left.A\right|_{P_{\sigma} \mathcal{K}}$ is a bounded operator.

\section{The PROPERTY (L) IN $\mathcal{K}_{J}$}

Our first main theorem is as follows.

Theorem 5. Let $\mathfrak{A}$ be a non-negative Hamiltonian operator on the Hilbert space $\mathcal{H}=\mathcal{G} \oplus \mathcal{G}$. If $\mathfrak{A} \in(\mathrm{L})$ in $\mathcal{K}_{J}$, then $\mathfrak{A}$ is bounded.

Proof. Instead of $\mathfrak{A}$ we consider the operator $\mathfrak{A}_{1}:=i \mathfrak{A}+i a J$ with $a>0$ and note that $\mathfrak{A}$ is bounded if and only if $\mathfrak{A}_{1}$ is bounded. Since $\mathfrak{A}$ is a Hamiltonian and $i J$ is self-adjoint in $\mathcal{K}_{\mathfrak{J}}, \mathfrak{A}_{1}$ is self-adjoint in $\mathcal{K}_{\mathfrak{J}}$. Claim: $\mathfrak{A}_{1}$ is a maximal uniformly dissipative operator in $\mathcal{K}_{J}$. Indeed, we have

$$
\operatorname{Im}\left(J \mathfrak{A}_{1} x, x\right)=\operatorname{Im}[i \mathfrak{A} x, x]_{J}+\operatorname{Im} i a(x, x) \geq a(x, x), \quad x \in \mathcal{H} .
$$

Hence $J \mathfrak{A}_{1}$ is a uniformly dissipative operator in the Hilbert space $\mathcal{H}$ and therefore $\lambda=0$ is a point of regular type of $J \mathfrak{A}_{1}$ (see [AI, p. 92]) and, since $J$ is unitary, also of $\mathfrak{A}_{1}$. Because self-adjoint operators in Krein spaces, such as $\mathfrak{A}_{1}$ in $\mathcal{K}_{\mathfrak{J}}$, have no real residual spectrum (see [L1] or [AI, Theorem 2.1.16]), $0 \in \rho\left(\mathfrak{A}_{1}\right)$. Hence $0 \in \rho\left(J \mathfrak{A}_{1}\right)$ and this inclusion is a (necessary and) sufficient condition for $J \mathfrak{A}_{1}$ to be a maximal uniformly dissipative operator in $\mathcal{H}$. Therefore $\mathfrak{A}_{1}$ is a maximal uniformly dissipative operator in $\mathcal{K}_{J}$. This completes the proof of the claim.

From the claim, the fact that $\mathfrak{A}_{1} \in(\mathrm{L})$ in $\mathcal{K}_{J}$, and Lemma 11, it follows that $\mathfrak{A}_{1}$ has only non-real spectrum, $\sigma_{+}\left(\mathfrak{A}_{1}\right):=\sigma\left(\mathfrak{A}_{1}\right) \cap \mathbb{C}^{+}$is a bounded set, and $\mathcal{L}_{+}:=P_{+}\left(\mathfrak{A}_{1}\right) \mathcal{H}$ is an $\mathfrak{A}_{1}$-invariant subspace which is maximal uniformly positive in $\mathcal{K}_{J}$.

There are two ways to proceed with the proof. We think that both are useful and interesting and therefore we present them both. 
I: We use Lemma 3 and follow some of the arguments in [AKK]. Consider the operator $-\mathfrak{A}_{1}^{*}$ where the adjoint is taken in $\mathcal{K}_{J}$. From the claim and $\mathrm{AI}$ Proposition 2.2.7, Proposition 2.2.32] it follows that $-\mathfrak{A}_{1}^{*}$ is also maximal uniformly dissipative in $\mathcal{K}_{J}$. Since $\mathfrak{A} \in(\mathrm{L})$, we have $\mathfrak{A}_{1} \in(\mathrm{L})$, and hence, according to Lemma 3 also $-\mathfrak{A}_{1}^{*} \in(\mathrm{L})$. Thus we may apply Lemma 1 to $-\mathfrak{A}_{1}^{*}$ and we conclude that $\sigma_{+}\left(-\mathfrak{A}_{1}^{*}\right):=\sigma\left(-\mathfrak{A}_{1}^{*}\right) \cap \mathbb{C}^{+}$is a bounded set and $\mathcal{M}_{+}:=P_{+}\left(-\mathfrak{A}_{1}^{*}\right) \mathcal{H}$ is an $\left(-\mathfrak{A}_{1}^{*}\right)-$ invariant maximal uniformly positive subspace in $\mathcal{K}_{J}$. From $\sigma_{+}\left(-\mathfrak{A}_{1}^{*}\right)=-\sigma_{+}\left(\mathfrak{A}_{1}\right)^{*}$ it follows that

$$
\begin{aligned}
P_{+}\left(-\mathfrak{A}_{1}^{*}\right) & =\frac{1}{2 \pi i} \int_{\lambda \in \Gamma_{+}\left(\sigma_{+}\left(-\mathfrak{A}_{1}^{*}\right)\right)}\left(\lambda+\mathfrak{A}_{1}^{*}\right)^{-1} d \lambda=\frac{1}{2 \pi i} \int_{\mu \in \Gamma_{+}\left(\sigma_{+}\left(\mathfrak{A}_{1}\right)\right)}\left(-\mu^{*}+\mathfrak{A}_{1}^{*}\right)^{-1} d \mu^{*} \\
& =\left(\frac{1}{2 \pi i} \int_{\mu \in \Gamma_{+}\left(\sigma_{+}\left(\mathfrak{A}_{1}\right)\right)}\left(\mu-\mathfrak{A}_{1}\right)^{-1} d \mu\right)^{*}=P_{+}\left(\mathfrak{A}_{1}\right)^{*} .
\end{aligned}
$$

Hence $\mathcal{M}_{+}=P_{+}\left(\mathfrak{A}_{1}\right)^{*} \mathcal{H}$. Its orthogonal complement $\mathcal{L}_{-}$in $\mathcal{K}_{J}$ equals

$$
\mathcal{L}_{-}=\operatorname{ker} P_{+}\left(\mathfrak{A}_{1}\right)=\left(I-P_{+}\left(\mathfrak{A}_{1}\right)\right) \mathcal{H}
$$

and is an $\mathfrak{A}_{1}$-invariant maximal uniformly negative subspace. Moreover, $\sigma\left(\left.\mathfrak{A}_{1}\right|_{\mathcal{L}_{-}}\right) \subset$ $\mathbb{C}^{-}$. Since $\sigma\left(\left.\mathfrak{A}_{1}\right|_{\mathcal{L}_{+}}\right) \subset \mathbb{C}^{+}$and $\mathfrak{A}_{1}$ is self-adjoint in $\mathcal{K}_{\mathfrak{J}}$, both $\mathcal{L}_{+}$and $\mathcal{L}_{-}$are neutral subspaces of $\mathcal{K}_{\mathfrak{J}}$ (see [AI, Corollary 2.3.11]) and

$$
\mathcal{H}=\mathcal{L}_{+}+\mathcal{L}_{-} .
$$

We can complete the proof in two directions:

Ia: From (7) and [AI, Proposition 1.1.25] it follows that $\mathcal{L}_{+}$and $\mathcal{L}_{-}$are in fact maximal neutral subspaces of $\mathcal{K}_{\mathfrak{J}}$. Because $\mathcal{L}_{+}=P_{+}\left(\mathfrak{A}_{1}\right) \mathcal{H}$ and $\mathfrak{A}_{1}$ is self-adjoint in $\mathcal{K}_{\mathfrak{J}}$, by Lemma 4 we have that $\mathfrak{A}_{1}$ is bounded.

Ib: From Lemma 2 with $\mathcal{K}_{1}=\mathcal{K}_{2}=\mathcal{K}_{\mathfrak{J}}, \mathcal{L}_{1}=\mathcal{G} \oplus\{0\}, \mathcal{M}_{1}=\{0\} \oplus \mathcal{G}, \mathcal{L}_{2}=\mathcal{L}_{+}$ and $\mathcal{M}_{2}=\mathcal{L}_{-}$, it follows that there exists a unitary operator $V$ on $K_{\mathfrak{J}}$ such that $V \mathcal{L}_{1}=\mathcal{L}_{2}$ and $V \mathcal{M}_{1}=\mathcal{M}_{2}$. The unitarity of $V$ implies that $\mathfrak{B}:=-i V^{-1} \mathfrak{A}_{1} V$ is a Hamiltonian operator. Since the summands in (7) are $\mathfrak{A}_{1}$-invariant, $\mathfrak{B}$ is a diagonal operator with respect to the decomposition (11). If we take $V$ as in (51), then with respect to this decomposition $\mathfrak{B}$ has the representation

$$
\mathfrak{B}=-i\left[\begin{array}{cc}
\left.V_{21}^{-1} \mathfrak{A}_{1}\right|_{\mathcal{L}_{+}} V_{21} & 0 \\
0 & -\left(\left.V_{21}^{-1} \mathfrak{A}_{1}\right|_{\mathcal{L}_{+}} V_{21}\right)^{*}
\end{array}\right] .
$$

Now observe that $\left.\mathfrak{A}_{1}\right|_{\mathcal{L}_{+}}$is a bounded operator (as $\mathcal{L}_{+}$is a Riesz subspace of $\mathfrak{A}_{1}$ ), hence the two non-zero entries in the representation are bounded. This implies the boundedness of $\mathfrak{B}$, or equivalently, the boundedness of $\mathfrak{A}_{1}$.

II: We use Lemma 4 directly. It suffices to show that $\mathcal{L}_{+}$is a maximal $\mathfrak{J}$-neutral subspace. For then $\mathfrak{A}_{1}$ satisfies the conditions of Lemma 4 and hence it is bounded. Let $x \oplus y \in \mathcal{L}_{+}$with $x, y \in \mathcal{G}$. Since $\mathcal{L}_{+}$is a uniformly positive subspace in $\mathcal{K}_{J}$, there is an $\varepsilon>0$ such that

$$
2 \operatorname{Re}(y, x) \geq \varepsilon((x, x)+(y, y)) .
$$

Hence on $\mathcal{G}$ there is a bounded and boundedly invertible operator $K$ such that $i K$ is a uniformly dissipative operator on $\mathcal{G}$ and $y=K x$. Since $\mathcal{L}_{+}$is a maximal uniformly positive subspace of $\mathcal{K}_{J}$, $\operatorname{dom} K=\mathcal{G}$ and $\operatorname{ran} K=\mathcal{G}$. On the other hand, $\mathcal{L}_{+}$is an invariant subspace of the self-adjoint operator $\mathfrak{A}_{1}$ in $\mathcal{K}_{\mathfrak{J}}$. Hence $\mathcal{L}_{+}$is a 
neutral subspace of $\mathcal{K}_{\mathfrak{J}}$ (see, [AI, Corollary 2.3.11]). This implies $\operatorname{Im}(K x, x)=0$. So,

$$
(K x, x)=\operatorname{Re}(K x, x) \geq \varepsilon(x, x),
$$

that is, $K$ is a uniformly positive operator on $\mathcal{G}$. Assume that $\mathcal{L}_{+}$is not a maximal neutral subspace in $\mathcal{K}_{\mathfrak{J}}$ and that it is properly contained in the neutral subspace $\widetilde{\mathcal{L}_{+}}$. Then $\widetilde{\mathcal{L}_{+}}$contains an element of the form $v \oplus 0$ with $0 \neq v \in \mathcal{G}$, and because $v \oplus K v \in \widetilde{\mathcal{L}_{+}}$also $(i v) \oplus K v \in \widetilde{\mathcal{L}_{+}}$. Since $\widetilde{\mathcal{L}_{+}}$is a neutral subspace in $\mathcal{K}_{\mathfrak{J}}$, we have $(K v, v)=0$ and hence $v=0$. This contradicts $v \neq 0$ and therefore $\mathcal{L}_{+}$is a maximal neutral subspace of $\mathcal{K}_{\mathfrak{J}}$.

\section{3. (L)-PROPERTY IN $\mathcal{K}_{\mathfrak{J}}$}

In the proof of Theorem 6 below we adapt some of the arguments Shkalikov used in his proof of Lemma 1$]$ see [S].

Theorem 6. Let $\mathfrak{A}$ be a non-negative Hamiltonian operator on the Hilbert space $\mathcal{H}=\mathcal{G} \oplus \mathcal{G}$. If $\mathfrak{A} \in(\mathrm{L})$ in $\mathcal{K}_{\mathfrak{J}}$, then $\mathfrak{A}$ is bounded.

Proof. As in the proof of Theorem $\left[5\right.$ we consider the operator $\mathfrak{A}_{1}=i \mathfrak{A}+i a J$ but now with $a>1$. It is maximal uniformly dissipative in $\mathcal{K}_{J}$. We claim that for all $a>1$,

$$
S_{1}:=\{\lambda=\alpha+i \beta \in \mathbb{C}|\alpha, \beta \in \mathbb{R},| \beta \mid \leq 1\} \subset \rho\left(\mathfrak{A}_{1}\right) .
$$

Indeed, for $\lambda=\alpha+i \beta$ in the strip $S_{1}$, the operator $\mathfrak{A}_{1}-\lambda$ is uniformly dissipative in $\mathcal{K}_{J}$, because

$$
\begin{aligned}
\operatorname{Im}\left[\left(\mathfrak{A}_{1}-\lambda\right) x, x\right]_{J} & =\operatorname{Im}\left[\mathfrak{A}_{1} x, x\right]_{J}+a(x, x)-\beta(J x, x) \\
& \geq(a-|\beta|)(x, x) \geq(a-1)(x, x) .
\end{aligned}
$$

With $\mathfrak{A}_{1}$ being maximal uniformly dissipative also $\mathfrak{A}_{1}-\lambda$ is maximal uniformly dissipative in $\mathcal{K}_{J}$. Hence $0 \in \rho\left(\mathfrak{A}_{1}-\lambda\right)$ for all $\lambda$ in the strip and this is equivalent to (8).

We shall show that

$$
\begin{aligned}
& \sigma_{+}\left(\mathfrak{A}_{1}\right):=\sigma\left(\mathfrak{A}_{1}\right) \cap \mathbb{C}^{+} \text {is a bounded set, } \\
& P_{+}\left(\mathfrak{A}_{1}\right) \mathcal{H} \text { is a maximal neutral subspace of } \mathcal{K}_{\mathfrak{J}} .
\end{aligned}
$$

Since $\mathfrak{A}_{1}$ is self-adjoint in $\mathcal{K}_{\mathfrak{J}}$ and (8) implies $\sigma\left(\mathfrak{A}_{1}\right) \cap \mathbb{R}=\emptyset$, the theorem follows from Lemma 4 and the proof is complete.

Proof of (9). Assume that the fundamental decomposition (2) for $\mathcal{K}=\mathcal{K}_{\mathfrak{J}}$ is such that $\mathcal{K}^{+}$is the maximal uniformly positive subspace contained in $\operatorname{dom} \mathfrak{A}_{1}$. We denote by $\mathfrak{J}_{1}$ the corresponding fundamental symmetry. With respect to the decomposition (2) the operators $\mathfrak{J}_{1}$ and $\mathfrak{A}_{1}$ have the matrix representations

$$
\mathfrak{J}_{1}=\left[\begin{array}{cc}
I & 0 \\
0 & -I
\end{array}\right], \quad \mathfrak{A}_{1}=\left[\begin{array}{cc}
A_{11} & -A_{21}^{*} \\
A_{21} & A_{22}
\end{array}\right] .
$$


Here the operators $A_{11}$ and $A_{21}$ are bounded and the first of these and $A_{22}$ are self-adjoint operators in the Hilbert spaces $\left\{\mathcal{K}^{+},[\cdot, \cdot]_{\mathfrak{J}}\right\}$ and $\left\{\mathcal{K}^{-},-[\cdot, \cdot]_{\mathfrak{J}}\right\}$, respectively. Hence for non-real $\lambda$ 's the operator functions

$$
\begin{aligned}
& G(\lambda)=-A_{21}^{*}\left(A_{22}-\lambda\right)^{-1}, \\
& F(\lambda)=\left(A_{22}-\lambda\right)^{-1} A_{21}, \\
& M(\lambda)=\left(I-G(\lambda) A_{21}\left(A_{11}-\lambda\right)^{-1}\right)\left(A_{11}-\lambda\right)
\end{aligned}
$$

are well defined and their values are bounded operators, and we have the block matrix factorization

$$
\mathfrak{A}_{1}-\lambda=\left[\begin{array}{cc}
I & G(\lambda) \\
0 & I
\end{array}\right] \times\left[\begin{array}{cc}
M(\lambda) & 0 \\
0 & A_{22}-\lambda
\end{array}\right] \times\left[\begin{array}{cc}
I & 0 \\
F(\lambda) & I
\end{array}\right] .
$$

This factorization implies that a non-real $\lambda$ belongs to $\rho\left(\mathfrak{A}_{1}\right)$ if and only if 0 belongs to $\rho(M(\lambda))$. To prove (9) it suffices to show

$$
\sigma_{+}\left(\mathfrak{A}_{1}\right) \subset\left(\mathbb{C}^{+} \backslash S_{1}\right) \cap D,
$$

where $D$ is the disk $|\lambda| \leq\left\|A_{11}\right\|+\left\|A_{21}\right\|^{2}$. If $\lambda \in \mathbb{C}^{+}$does not belong to the set on the right, then either $\lambda \in S_{1}$ or $\operatorname{Im} \lambda>1$ and $|\lambda|>\left\|A_{11}\right\|+\left\|A_{21}\right\|^{2}$. In the first case, by (8), $\lambda \in \rho\left(\mathfrak{A}_{1}\right)$. In the second case

$$
\left\|G(\lambda) A_{21}\left(A_{11}-\lambda\right)^{-1}\right\| \leq \frac{1}{\operatorname{Im} \lambda} \frac{\left\|A_{21}\right\|^{2}}{|\lambda|-\left\|A_{11}\right\|}<1,
$$

which implies that $0 \in \rho(M(\lambda))$, that is, then also $\lambda \in \rho\left(\mathfrak{A}_{1}\right)$. Hence in either case $\lambda \notin \sigma_{+}\left(\mathfrak{A}_{1}\right)$. This proves (11) and completes the proof of (9).

Proof of (10). Denote by $P_{+}\left(\mathfrak{A}_{1}\right)$ the Riesz projection relative to $\sigma_{+}\left(\mathfrak{A}_{1}\right)$. Since $\mathfrak{A}_{1}$ is a self-adjoint operator in $\mathcal{K}_{\mathfrak{J}}$, by [AI, Corollary 2.3.11]), $\mathcal{L}_{+}:=P_{+}\left(\mathfrak{A}_{1}\right) \mathcal{K}$ is a neutral subspace in $\mathcal{K}_{\mathfrak{J}}$. Hence there is a bounded operator $K:\left\{\mathcal{K}^{+},[\cdot, \cdot]_{\mathfrak{J}}\right\} \rightarrow$ $\left\{\mathcal{K}^{-},-[\cdot, \cdot]_{\mathfrak{J}}\right\}$, called the angular operator for $\mathcal{L}_{+}$, such that with respect to the fundamental decomposition (2) of $\mathcal{K}_{\mathfrak{J}}$

$$
\mathcal{L}_{+}=\left\{x=x^{+}+K x^{+} \mid x^{+} \in P^{+} \mathcal{L}_{+}\right\} .
$$

This subspace is maximal if and only if $P^{+} \mathcal{L}_{+}=\mathcal{K}^{+}$, where $P^{+}$is the orthogonal projection in $\mathcal{K}_{\mathfrak{J}}$ onto $\mathcal{K}^{+}$. With respect to the fundamental decomposition (2) the operator $P_{+}\left(\mathfrak{A}_{1}\right)$ has the representation

$$
P_{+}\left(\mathfrak{A}_{1}\right)=\left[\begin{array}{cc}
P & Q \\
K P & K Q
\end{array}\right],
$$

where $P$ and $Q$ are bounded operators. Consequently, for the maximality of $\mathcal{L}_{+}$it is sufficient to show that $0 \in \rho(P)$ and for this to hold it suffices to prove

$$
\operatorname{Re}\left[P x^{+}, x^{+}\right]_{\mathfrak{J}} \geq \frac{1}{2}\left(x^{+}, x^{+}\right) \text {for all } x^{+} \in \mathcal{K}^{+} .
$$

The proof makes use of an approximation argument. We consider the auxiliary operator $\mathfrak{B}_{b}:=\mathfrak{A}_{1}+i b \mathfrak{J}_{1}$ with $0<b<\frac{a-1}{\left\|\mathfrak{J}_{1}\right\|}$ and let $b \downarrow 0$. Here the norm is computed in the inner product of the Hilbert space $\mathcal{H}$. The proof is divided into several steps. We shall show

(a) $\mathfrak{B}_{b}$ is maximal uniformly dissipative in $\mathcal{K}_{\mathfrak{J}}$ and

(b) $\mathfrak{B}_{b} \in(\mathrm{L})$ in $\mathcal{K}_{\mathfrak{J}}$, 
so that, by Lemma $1 P_{+}\left(\mathfrak{B}_{b}\right)$ exists, and then we show

(c) $P_{+}\left(\mathfrak{B}_{b}\right) \rightarrow P_{+}\left(\mathfrak{A}_{1}\right)$ as $b \downarrow 0$ and

(d) $\operatorname{Re}\left[P_{+}\left(\mathfrak{B}_{b}\right) x^{+}, x^{+}\right]_{\mathfrak{J}} \geq \frac{1}{2}\left(x^{+}, x^{+}\right), x^{+} \in \mathcal{K}^{+}$, from which, since $P=P^{+} P_{+}\left(\mathfrak{A}_{1}\right) P^{+}$, (13) follows.

(a) For $x \in \mathcal{H}$,

$$
\operatorname{Im}\left[\mathfrak{B}_{b} x, x\right]_{\mathfrak{J}}=\operatorname{Im}\left[\mathfrak{A}_{1} x, x\right]_{\mathfrak{J}}+\operatorname{Im} i b\left[\mathfrak{J}_{1} x, x\right]_{\mathfrak{J}}=b\left[\mathfrak{J}_{1} x, x\right]_{\mathfrak{J}},
$$

and hence, since $\left[\mathfrak{J}_{1} \cdot, \cdot\right]_{\mathfrak{J}}$ is a Hilbert space inner product on $\mathcal{K}_{\mathfrak{J}}, \mathfrak{B}_{b}$ is uniformly dissipative in $\mathcal{K}_{\mathfrak{J}}$. Hence $\lambda=0$ is a point of regular type for $\mathfrak{B}_{b}$. The analog of (14) for $-\mathfrak{B}_{b}^{*}=-\mathfrak{A}_{1}+i b \mathfrak{J}_{1}$ also holds, hence $-\mathfrak{B}_{b}^{*}$ is also uniformly dissipative in $\mathcal{K}_{\mathfrak{J}}$ and therefore $\lambda=0$ is also a point of regular type for $\mathfrak{B}_{b}^{*}$. This implies that $\lambda=0$ is in fact a regular point for $\mathfrak{B}_{b}$ (and for $\mathfrak{B}_{b}^{*}$; because, if it is not a regular point for one, it would be an eigenvalue for the other). This implies (a).

(b) This follows directly from the fact that with $\mathfrak{A} \in(\mathrm{L})$ also $\mathfrak{A}_{1} \in(\mathrm{L})$ in the space $\mathcal{K}_{\mathfrak{J}}$. Applying Lemma 1 we obtain

$$
\begin{aligned}
& \sigma_{+}\left(\mathfrak{B}_{b}\right):=\sigma\left(\mathfrak{B}_{b}\right) \cap \mathbb{C}^{+} \text {is a bounded set and } \\
& P_{+}\left(\mathfrak{B}_{b}\right) \mathcal{H} \text { is a maximal uniformly positive subspace of } \mathcal{K}_{\mathfrak{J}} .
\end{aligned}
$$

(c) With respect to the decomposition (2) $\mathfrak{B}_{b}$ has the matrix representation

$$
\mathfrak{B}_{b}=\left[\begin{array}{cc}
A_{11}+i b & -A_{21}^{*} \\
A_{21} & A_{22}-i b
\end{array}\right] .
$$

Hence for $\lambda \in \mathbb{C}^{+}$with $|\lambda|$ large we have

$$
\mathfrak{B}_{b}-\lambda=\left[\begin{array}{cc}
I & G_{b}(\lambda) \\
0 & I
\end{array}\right] \times\left[\begin{array}{cc}
M_{b}(\lambda) & 0 \\
0 & A_{22}-i b-\lambda
\end{array}\right] \times\left[\begin{array}{cc}
I & 0 \\
F_{b}(\lambda) & I
\end{array}\right],
$$

where

$$
\begin{aligned}
& G_{b}(\lambda)=-A_{21}^{*}\left(A_{22}-i b-\lambda\right)^{-1}, \\
& F_{b}(\lambda)=\left(A_{22}-i b-\lambda\right)^{-1} A_{21}, \\
& M_{b}(\lambda)=\left(I-G_{b}(\lambda) A_{21}\left(A_{11}+i b-\lambda\right)^{-1}\right)\left(A_{11}+i b-\lambda\right) .
\end{aligned}
$$

As in the beginning of this proof but now with the help of

$$
\operatorname{Im}\left[\mathfrak{B}_{b} x, x\right]_{J}=\operatorname{Im}\left[\mathfrak{A}_{1} x, x\right]_{J}+\operatorname{Im} i b\left[\mathfrak{J}_{1} x, x\right]_{J} \geq\left(a-b\left\|\mathfrak{J}_{1}\right\|\right)(x, x), x \in \mathcal{H},
$$

and the fact that $a-b\left\|\mathfrak{J}_{1}\right\|>1$, it can be shown that (8) also holds for $\mathfrak{B}_{b}$, that is, $S_{1} \subset \rho\left(\mathfrak{B}_{b}\right)$. (Note: Above we showed that $\mathfrak{B}_{b}$ is uniformly dissipative in $\mathcal{K}_{\mathfrak{J}}$, but by (17), $\mathfrak{B}_{b}$ is also uniformly dissipative in $\mathcal{K}_{J}$.) Moreover, by the same arguments as above for $\mathfrak{A}_{1}$, we find that

$$
\sigma_{+}\left(\mathfrak{B}_{b}\right) \subset\left(\mathbb{C}^{+} \backslash S_{1}\right) \cap D_{s},
$$

where $D_{s}$ is the closed disk around the origin with radius $s=\left\|A_{21}\right\|^{2}+\left\|A_{11}+i b\right\|$. We leave the details of the proof to the reader. Hence for $r>\left\|A_{21}\right\|^{2}+\left\|A_{11}\right\|+\frac{a-1}{\left\|\mathfrak{J}_{1}\right\|}$, the contour

$$
\Gamma_{r}:=[-r, r] \cup\left\{r e^{i \varphi} \mid \varphi \in[0, \pi]\right\},
$$

which we assume to be positively oriented, encircles $\sigma_{+}\left(\mathfrak{A}_{1}\right)$ and $\sigma_{+}\left(\mathfrak{B}_{b}\right)$ for all $b \in\left(0, \frac{a-1}{\left\|\mathfrak{J}_{1}\right\|}\right)$. The other parts of the spectra of $\mathfrak{A}_{1}$ and $\mathfrak{B}_{b}$ are contained in the exterior of $\Gamma_{r}$. Set

$$
m_{r}=\max \left\{\left\|\left(\mathfrak{A}_{1}-\lambda\right)^{-1}\right\| \mid \lambda \in \Gamma_{r}\right\}
$$


Then for $\lambda \in \Gamma_{r}$ and sufficiently small $b$,

$$
\left\|\left(\mathfrak{B}_{b}-\lambda\right)^{-1}-\left(\mathfrak{A}_{1}-\lambda\right)^{-1}\right\| \leq \frac{b m_{r}^{2}\left\|\mathfrak{J}_{1}\right\|}{1-b m_{r}\left\|\mathfrak{J}_{1}\right\|} .
$$

Therefore

$$
\left\|P_{+}\left(\mathfrak{B}_{b}\right)-P_{+}\left(\mathfrak{A}_{1}\right)\right\| \rightarrow 0 \text { as } b \downarrow 0 .
$$

This completes the proof of (c).

(d) We first observe that for $M_{b}(\lambda)$ in (16)

$$
\lim _{r \rightarrow \infty} r e^{i \varphi} M_{b}\left(r e^{i \varphi}\right)^{-1}=-I,
$$

uniformly in $\varphi \in[0, \pi]$. This follows from

$$
\begin{aligned}
& r e^{i \varphi} M_{b}\left(r e^{i \varphi}\right)^{-1} \\
& =r e^{i \varphi}\left(A_{11}+i b-r e^{i \varphi}\right)^{-1}\left\{I+A_{21}^{*}\left(A_{22}-i b-r e^{i \varphi}\right)^{-1} A_{21}\left(A_{11}+i b-r e^{i \varphi}\right)^{-1}\right\}^{-1},
\end{aligned}
$$

and the limits, uniformly in $\varphi \in[0, \pi]$,

$$
\begin{gathered}
\lim _{r \rightarrow \infty} r e^{i \varphi}\left(A_{11}+i b-r e^{i \varphi}\right)^{-1}=-I, \\
\lim _{r \rightarrow \infty}\left(I+A_{21}^{*}\left(A_{22}-i b-r e^{i \varphi}\right)^{-1} A_{21}\left(A_{11}+i b-r e^{i \varphi}\right)^{-1}\right)=I .
\end{gathered}
$$

The last limit is valid because of the estimate

$$
\left\|\left(A_{22}-i b-r e^{i \varphi}\right)^{-1}\right\| \leq \frac{1}{b}
$$

the boundedness of $A_{21}$, and the limit, uniformly in $\varphi \in[0, \pi]$,

$$
\lim _{r \rightarrow \infty}\left(A_{11}+i b-r e^{i \varphi}\right)^{-1}=0 .
$$

The operator $P_{+}\left(\mathfrak{B}_{b}\right)$ can be rewritten as

$$
P_{+}\left(\mathfrak{B}_{b}\right)=-\frac{1}{2 \pi i} \int_{-r}^{r}\left(\mathfrak{B}_{b}-\alpha\right)^{-1} d \alpha+\frac{1}{2 \pi} \int_{0}^{\pi}\left(I-\frac{e^{-i \varphi}}{r} \mathfrak{B}_{b}\right)^{-1} d \varphi .
$$

From the property that $\mathfrak{B}_{b}$ is dissipative in $\mathcal{K}_{\mathfrak{J}}$ we have

$$
\operatorname{Re}\left(-\frac{1}{2 \pi i} \int_{-r}^{r}\left(\mathfrak{B}_{b}-\alpha\right)^{-1} d \alpha\right)=-\frac{1}{2 \pi} \operatorname{Im} \int_{-r}^{r}\left(\mathfrak{B}_{b}-\alpha\right)^{-1} d \alpha \geq 0 .
$$

We now obtain as $r \rightarrow \infty$,

$$
\begin{aligned}
& \operatorname{Re}\left[P_{+}\left(\mathfrak{B}_{b}\right) x^{+}, x^{+}\right]_{\mathfrak{J}} \\
& \geq \operatorname{Re} \frac{1}{2 \pi} \int_{0}^{\pi}\left[\left(I-\frac{e^{-i \varphi}}{r} \mathfrak{B}_{b}\right)^{-1} x^{+}, x^{+}\right]_{\mathfrak{J}} d \varphi \quad \text { by (19) and (20) } \\
& =-\operatorname{Re} \frac{1}{2 \pi} \int_{0}^{\pi}\left[r e^{i \varphi} M_{b}\left(r e^{i \varphi}\right)^{-1} x^{+}, x^{+}\right]_{\mathfrak{J}} d \varphi \quad \text { by (15) } \\
& \rightarrow \frac{1}{2}\left[x^{+}, x^{+}\right]_{\mathfrak{J}}=\frac{1}{2}\left(x^{+}, x^{+}\right), \quad \text { by }(18)
\end{aligned}
$$

and this proves (d) and completes the proof of the theorem. 


\section{EXAMPLES}

First we give an example which shows that in the assumptions of Theorem 5 it is essential that the operator $\mathfrak{A}$ on the Hilbert space $\mathcal{G} \oplus \mathcal{G}$ satisfies the condition (L).

Example 7. Let $A: \mathcal{G} \rightarrow \mathcal{G}$ be an unbounded self-adjoint uniformly positive operator on $\mathcal{G}$. Then the operator

$$
\mathfrak{A}=\left[\begin{array}{cc}
A & 0 \\
0 & -A
\end{array}\right]
$$

is a non-negative Hamiltonian operator, which is unbounded.

The following example shows that in Theorem 6 it is essential that the Hamiltonian operator $\mathfrak{A}$ is non-negative.

Example 8. Let $A$ be an unbounded self-adjoint operator on $\mathcal{G}$ and define the operator $\mathfrak{A}$ by

$$
\mathfrak{A}=\left[\begin{array}{cc}
I & -i \\
0 & I
\end{array}\right] \times\left[\begin{array}{cc}
0 & 0 \\
A & 0
\end{array}\right] \times\left[\begin{array}{cc}
I & -i \\
0 & I
\end{array}\right] .
$$

Evidently, $\operatorname{dom} \mathfrak{A}=\{x \oplus y \mid x, y \in \mathcal{G}, x-i y \in \operatorname{dom} A\}$. Since both operators

$$
\left[\begin{array}{cc}
I & -i \\
0 & I
\end{array}\right], \quad i\left[\begin{array}{ll}
0 & 0 \\
A & 0
\end{array}\right]
$$

are self-adjoint in $\mathcal{K}_{\mathfrak{J}}$ and the first is bounded and boundedly invertible, the operator $\mathfrak{A}$ is Hamiltonian. On $(\operatorname{dom} A) \oplus(\operatorname{dom} A)$ the operator $\mathfrak{A}$ coincides with the operator

$$
\left[\begin{array}{cc}
-i A & -A \\
A & -i A
\end{array}\right]
$$

Hence $\mathfrak{A}$ is unbounded. For $x, y \in \operatorname{dom} A$ we have

$$
\operatorname{Im}[\mathfrak{A}(x \oplus y), x \oplus y]_{J}=2 \operatorname{Re}(A x, y),
$$

and hence the Hamiltonian operator $\mathfrak{A}$ is not non-negative. The subspace

$$
\mathcal{K}^{+}=\{i x \oplus x \mid x \in \mathcal{G}\}
$$

is maximal uniformly positive in $\mathcal{K}_{\mathfrak{J}}$, in fact $\mathcal{K}^{+}=P^{+} \mathcal{H}$, where $P^{+}=\frac{1}{2}(I+\mathfrak{J})$. From the definition (21) it follows that $\mathcal{K}^{+} \subset \operatorname{dom} \mathfrak{A}$, but the Hamiltonian operator $\mathfrak{A}$ is unbounded.

\section{REFERENCES}

[AI] T.YA. AzIzov AND I.S. IokHVIDOv, Foundations of the theory of linear operators in spaces with an indefinite metric, Nauka, Moscow, 1986 (Russian); English transl.: Linear operators in spaces with an indefinite metric, Wiley, New York, 1989. MR 90j:47042

[AKK] T.YA. AzIzov, V.K. KIRIAKIDI, AND G.A. KuRINA, An indefinite approach to the reduction of a non-negative Hamiltonian operator function to a block diagonal form, Funct. Anal. and Appl., 35 (3), 2001, 73-75 (Russian).

[B] M.L. Brodskii, On the properties of an operator mapping into itself the non-negative part of a space with an indefinite metric, Uspekhi Mat. Naut 14 (1), 1959, 147-152 (Russian). MR 21:5145

[DR] M.A. Dritschel, J. Rovnyak, Extension theorems for contraction operators on Krein spaces, Operator Theory: Adv. Appl., vol. 47, Birkhäuser Verlag, Basel, 1990, 221-305. MR 92m:47068 
[KL] M.G. KReIn AND H. LANGeR, On some mathematical principles in the linear theory of damped oscillations of continua, Proc. Int. Sympos. on Applications of the Theory of Functions in Continuum Mechanics, Tbilisi, 1963, Vol. II: Fluid and Gas Mechanics, Math. Methods, Moscow, 1965, 283-322 (Russian); English transl.: Integral Equations Operator Theory 1, 1978, 364-399 and 539-566. MR 80d:47026; MR 80h:47022

[L1] H. LANGeR, Zur Spektraltheorie J-selbstadjungierter Operatoren, Math. Ann. 146 (1), 1962, 60-85. MR 25:1450

[L2] H. Langer, Eine Verallgemeinerung eines Satzes von L.S. Pontrjagin, Math. Ann. 152 (5), 1963, 434-436. MR 28:1492

[LT] H. Langer And Chr. Tretter, Spectral decomposition of some nonselfadjoint block operator matrices, J. of Operator Theory 39, 1998, 339-359. MR 99d:47004

[S] A.A. ShKalikov, On the existence of invariant subspaces of dissipative operators in an inner product space, Fund. and Appl. Math. 5 (2), 1999, 627-635 (Russian). MR 2001k:47051

[Shm] Yu.L. Shmul'yan, Division in the class of J-expansive operators, Mat. Sb. (N.S.) 74 (116), 1967, 516-525 (Russian); English transl.: Math. USSR-Sbornik 3, 1967, 471-479.

Department of Mathematics, Voronezh State University, 394693 Voronezh, Russia

E-mail address: azizov@tom.vsu.ru

Department of Mathematics, University of Groningen, P.O. Box 800, 9700 AV GroninGEN, THE NETHERLANDS

E-mail address: dijksma@math.rug.nl

Department of Mathematics, Voronezh State University, 394693 Voronezh, Russia 